\title{
RL-SARSA Machine Learning Based Analog Radio over Fiber System
}

\author{
Muhammad Usman Hadi ${ }^{1}$ \\ ${ }^{1}$ Department of Electronic and Information Engineering, University of Bologna, Italy; \\ usmanhadi@ieee.org
}

\begin{abstract}
We propose a 10-Gb/s 64-quadrature amplitude modulation (QAM) signal-based Radio over Fiber (RoF) system for $50 \mathrm{~km}$ of standard single mode fiber length which utilizes Reinforcement Learning (RL) SARSA based decision method to indicate an effective decision which mitigates nonlinearity. By utilizing RL-SARSA algorithm, the results demonstrate that significant reduction can be obtained in terms of bit error rate.
\end{abstract}

Keywords: Radio over Fiber, Nonlinearities Mitigation, Reinforcement Learning (RL) method

\section{INTRODUCTION}

With advancement in next generation and 5G technology, the need of wireless distribution services is increasing manifold. We are surrounded in our lives by Internet of Things and therefore the connectivity from the base station (BS) to remote antenna heads (RAH) is very crucial. Radio over Fiber technology has been employed in different scenarios ranging from inhouse to outdoor applicative scenarios [1-4]. The enhancement of capacity and wireless coverage has posed significant challenges to the existing optical and wireless access networks. Cloud radio access network (C-RAN) architecture is one of the key enablers to achieve these goals. The 5G C-RAN should be able to control the centralized base band units (BBU) coming from many base stations and remote radio heads (RRHs). The interconnectivity of BBUs with RRHs is economically coherent with the distribution network known as the 'fronthaul' as shown in Fig. 1. Since, A-RoF systems can be considered as a cloud radio access network (C-RAN) interlinking the BBU and RRHs, therefore, this interlinking of BBUs with RRHs through distribution network is referred as the optical fronthaul [5]. A-RoF is a good candidate for such fronthaul applications.

While comparing A-RoF systems to Digital RoF systems [6-7] and Sigma Delta RoF systems [810], A-RoF systems are the most viable and economic links, however, they are liable to signal impairments and non-perfect behaviour of electro optical (EO) and optical electrical (OE) components in the A-RoF link. 


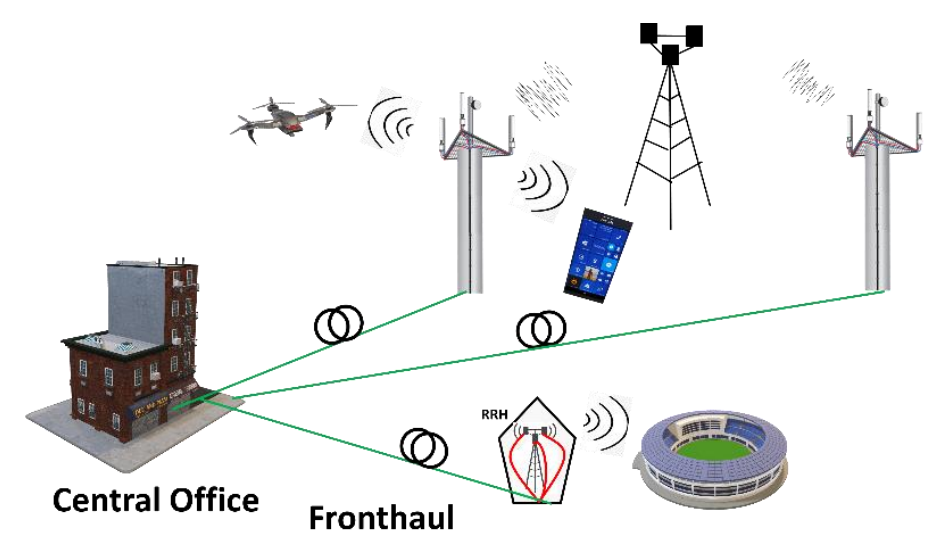

Fig. 1. Basic C-RAN architecture showing optical fronthaul.

The reduction of nonlinearities has gained immense importance to increase the optical system capacity. Nonlinearities mitigation has been proposed via Optical and Electrical domain methods, with latter getting more attention. Digital Predistortion has gained immense attention and therefore it is still looked to be an important methodology [11-16]. However, these DPD based linearization techniques are not so straight forward and are rather complex, time consuming and adds extra over heads [17]. Therefore. reducing such nonlinearities in A-RoF links effectively is still a bottle neck in efficient deployment of A-RoF systems.

With increasing trend of machine learning (ML) methods, mitigating the nonlinearities in optical communication systems with ML based techniques has become very popular. It was shown recently that mitigation of nonlinearities in A-RoF systems can be done using Support Vector Machine (SVM) based ML methods. Therefore, it will be interesting to continue the research in the same direction by exploiting other ML methods.

In this paper, a simulation study is presented for the mitigation of nonlinear impairments by employing Reinforcement Learning (RL) based machine learning methods. In the proposed system, 10-Gb/s with 64 quadrature amplitude modulation (QAM) signal is injected into distributed feedback (DFB) laser for $50 \mathrm{~km}$ single mode fiber transmission. It causes signal to suffer from nonlinearities due to opto-electronic devices in the RoF link as the ideal decision boundary is no longer linear. The utilization of RL-SARSA method is compared with the conventional decision method to mitigate the nonlinearities of the RoF system. 


\section{Experimental Setup}

The experimental setup utilized is demonstrated in Fig. 2. The arbitrary waveform generator (AWG) operating at 10-GSamples/s drives Distributed Feedback (DFB) Laser. The modulated 64-QAM signal is used while the laser diode (LD) is working at $1550 \mathrm{~nm}$. The Single Mode Fiber (SMF) of $50 \mathrm{~km}$ length is followed by variable optical attenuator referred as VOA whose functionality is to adjust the received optical power. The signal is photo detected by a photodiode having $0.7 \mathrm{~A} / \mathrm{W}$ of responsivity and $9.6 \mathrm{GHz}$ bandwidth. Then, the signal is passed through the digital processing block for machine learning decisions in offline manner. Here, the RL_SARSA is utilized that finds the optimum decision boundary. Minimal optimization algorithm is used to find the boundary condition while the training threshold is at about $5.1 \%$ with 1400 symbols to automatically obtain the optimal parameters. Finally, after this adjustment, parameter evaluation is done and compared to see if RL-SARSA based ML method is improving the performances of the RoF link considered or not.

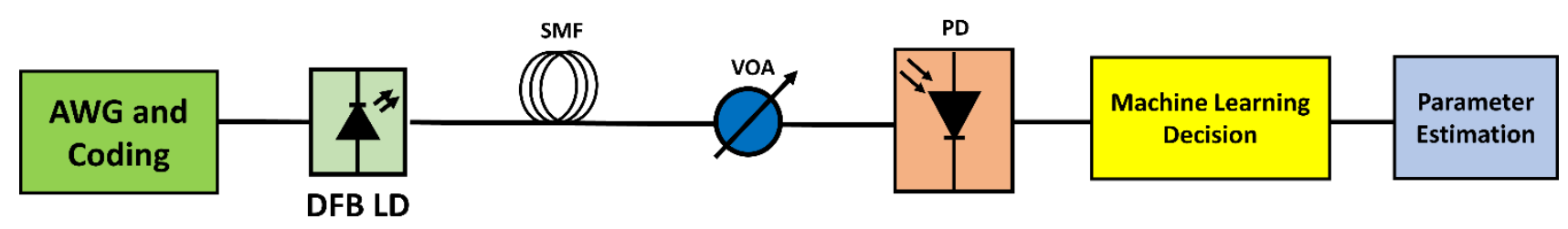

Fig. 2. Experimental setup. AWG: Arbitrary Waveform Generator, DFB laser: Distributed Feedback laser, VOA: Variable Optical Attenuator, SMF: Single Mode Fiber

$\mathrm{RL}$ is the self-learning scheme of machine learning which trains the system for intelligent decisions. The RL agents communicate directly with the environment by taking an action and receive feedback as a reward or penalty of that action. These feedbacks indicate the quality of actions to accomplish a specific job. Since, RL is ought to adopt within the unknown environment. In our proposed scenario, RL agents (RoF link) is lack of plenary knowledge of their environment. Therefore, SARSA, a well-known temporal difference (TD) algorithm is working on the basis of action-value function. The details of our proposed RL-SARSA algorithm can be found in [18].

\section{Experimental Results and Discussion}

The experimental results are discussed in this section where RL-SARSA based method is compared with conventional decision method is employed. Fig. 3 represents the bit error rate (BER) versus number of training data points for conventional and RL-SARSA method. The ML guided decision results in better reduction in nonlinearities as compared to conventional decision method. 


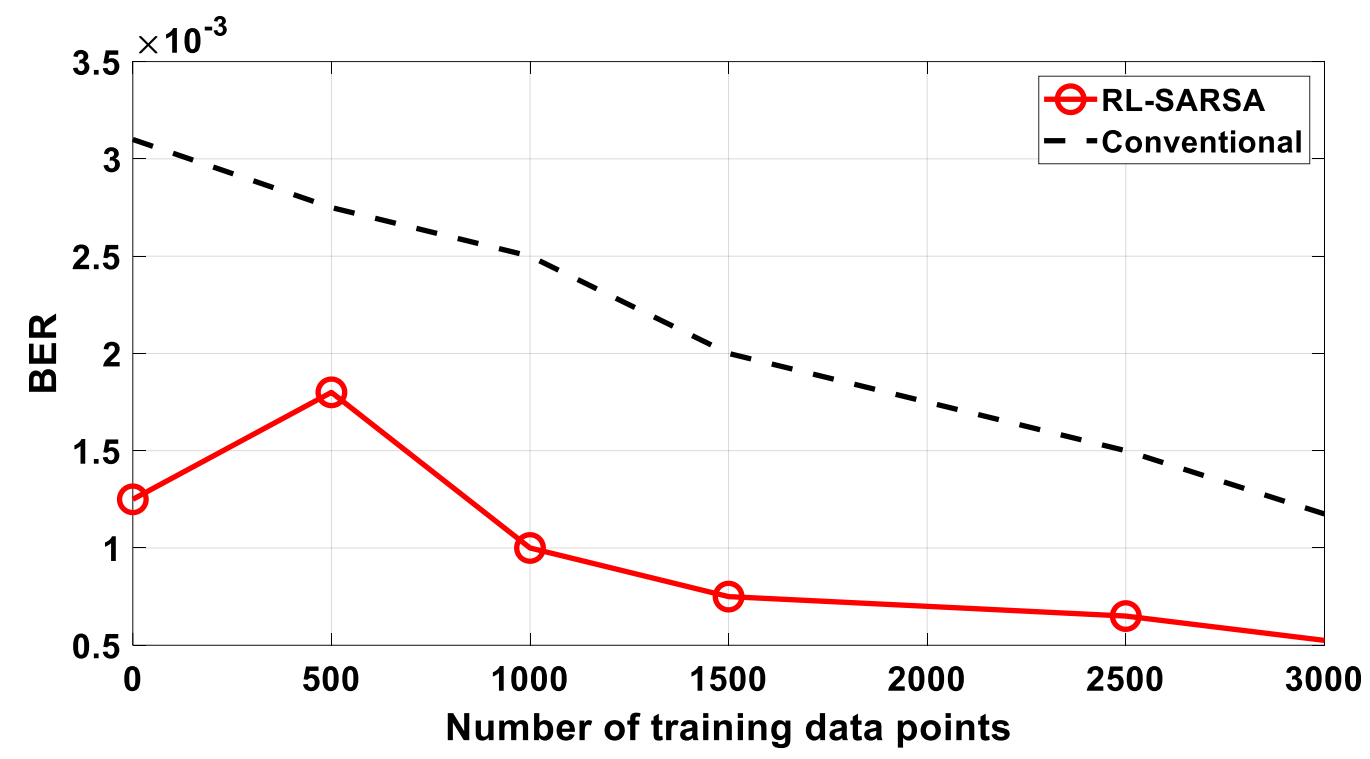

Fig. 3. BER vs number of training data points for RL-SARSA vs conventional

Then, Fig. 4 BER is shown with respect to RF input power which goes inside the $50 \mathrm{~km}$ fiber. By increasing the input power, the optical signal to noise ratio (OSNR) of the signal received is prohibitively large that results in a higher BER. The results show that RL-SARSA learning decision results in lower BER as compared to conventional decision method. This shows that RL-SARSA results in better decision.

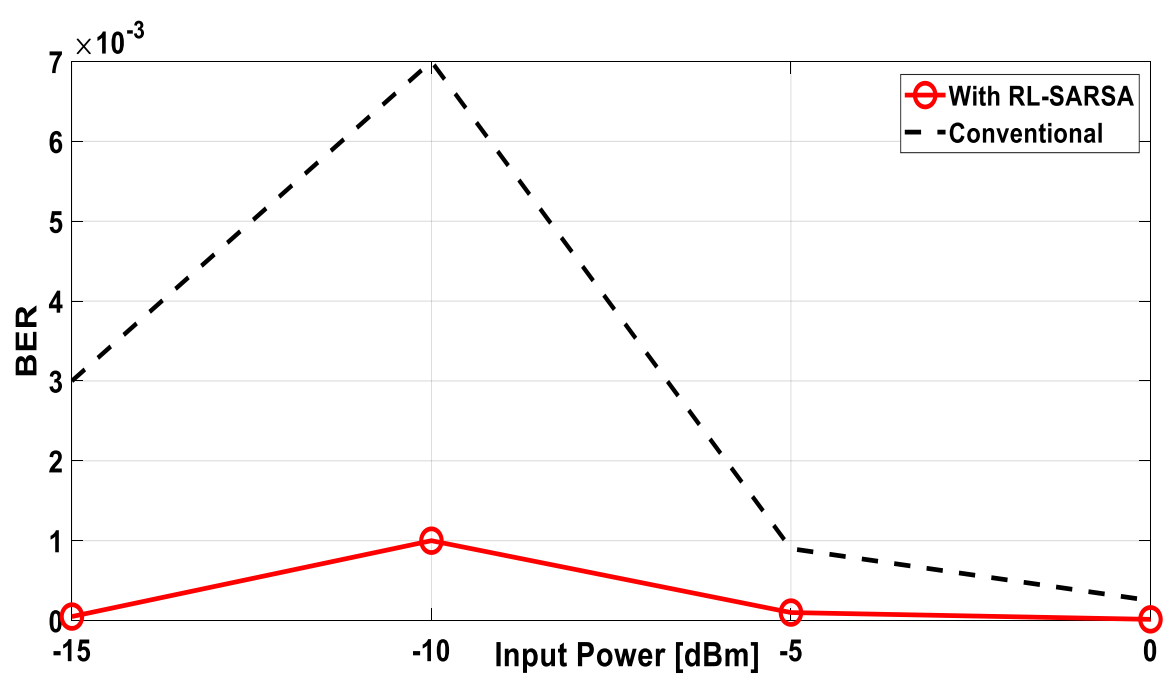

Fig. 4. BER vs launched input signal power for RL-SARSA vs. conventional decision method.

In Fig. 5, optical received power vs BER is shown. RL-SARSA decision shows that conventional method BER is brought down w.r.t received optical power. This shows that the proposed ML method has a vast improvement. 


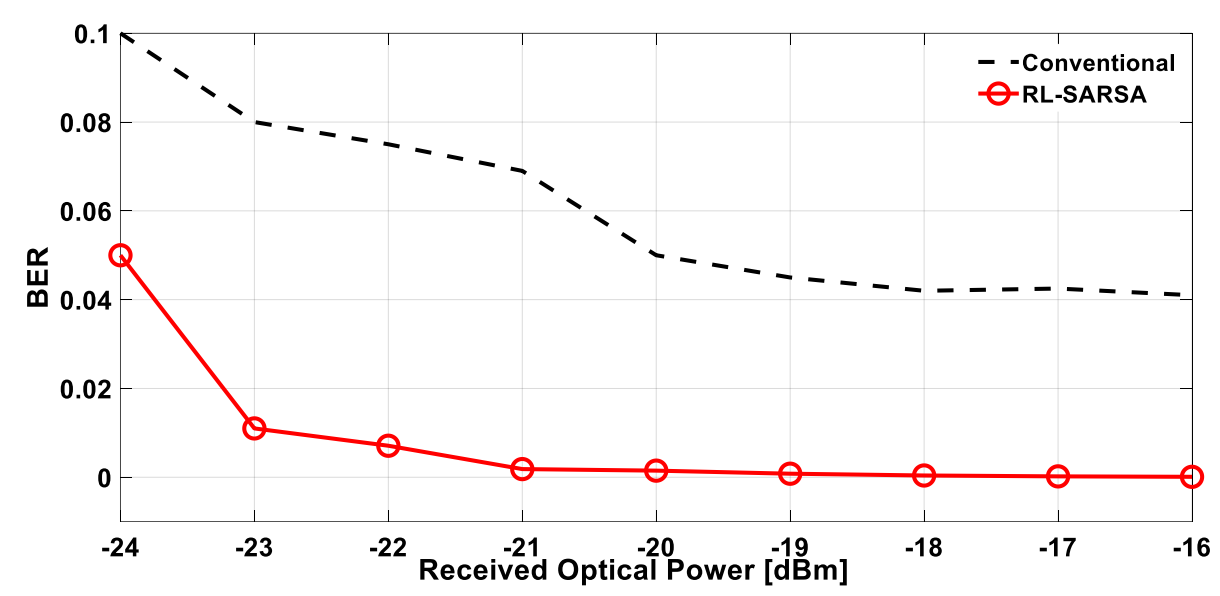

Fig. 5. BER vs received optical power for RL-SARSA vs. conventional decision method.

\section{CONCLUSION}

The article demonstrated RL-SARSA signal decision approach for reducing A- RoF system. The preliminary simulation results show that for a complex and high modulation format such $\mathrm{s}$ 256 QAM at $50 \mathrm{~km}$ of fiber length. RL-SARSA based ML decision results in significant reduction of the bit error rate in considered RoF system. The future work includes the simplification of algorithm and comparison with SVM, KNN and other methods envisages the use of other classifiers that can alleviate the impairments in the RoF system.

\section{REFERENCES}

[1] Gupta A., et al.A survey of 5G network: Architecture and emerging technologies, IEEE Access, 3 (2015), pp. 1206-1232 [2]

[2] China Mobile Research Institute, Beijing, China, C-RAN: the Road Towards Green RAN, White Paper (2013).

[3] Tartarini, G. and Faccin, P. (2005), Efficient characterization of harmonic and intermodulation distortion effects in dispersive radio over fiber systems with direct laser modulation. Microw. Opt. Technol. Lett., 46: 114-117. doi:10.1002/mop.20917

[4] D. Visani et al., "Wired and wireless multi-service transmission over 1mm-core GI-POF for in-home networks," in Electronics Letters, vol. 47, no. 3, pp. 203-205, 3 February 2011. doi: 10.1049/el.2010.7273

[5] I. A. Alimi, A. L. Teixeira and P. P. Monteiro, "Toward an Efficient C-RAN Optical Fronthaul for the Future Networks: A Tutorial on Technologies, Requirements, Challenges, and Solutions," in IEEE Communications Surveys \& Tutorials, vol. 20, no. 1, pp. 708-769, Firstquarter 2018. doi: 10.1109/COMST.2017.2773462

[6] Muhammad Usman Hadi, Hyun Jung, Salman Ghaffar, Pier Andrea Traverso, Giovanni Tartarini, Optimized digital radio over fiber system for medium range communication, Optics 
Communications, Volume 443, 2019, Pages 177-185, ISSN 0030-4018, https://doi.org/10.1016/j.optcom.2019.03.037.

[7] Hadi, M., Jung, H., Traverso, P., et al. (2019). Digital Radio Frequency Transport over Optical Fiber for 5G Fronthaul Links. Journal of Optical Communications, doi:10.1515/joc2019-0051

[8] Hadi M, Aslam N, Jung H. (2019). Performance appraisal of sigma delta modulated radio over fiber system. Journal of Optical Communications, doi:10.1515/joc-2018-0227. [9] Hadi, M., Hadi, M., Aslam, N., et al. (2019). Experimental Demonstration of MASH Based Sigma Delta Radio over Fiber System for 5G C-RAN Downlink. Journal of Optical Communications, doi:10.1515/joc-2019-0011

[10] Muhammad Usman Hadi, Pier Andrea Traverso, Giovanni Tartarini, Hyun Jung, Experimental characterization of Sigma Delta Radio over fiber system for 5G C-RAN downlink, ICT Express, 2019, ISSN 2405-9595, https://doi.org/10.1016/j.icte.2019.06.002. [11] Hadi, M.U., Nanni, J., Polleux, JL. et al. Opt Quant Electron (2019) 51: 205. https://doi.org/10.1007/s11082-019-1923-8

[12] M. U. Hadi, "Extending the benefits of LTE to unlicensed spectrum," 2015 International Conference on Information and Communication Technologies (ICICT), Karachi, 2015, pp. 13. doi: 10.1109/ICICT.2015.7469592

[13] M. U. Hadi et al., "Experimental evaluation of digital predistortion for VCSEL-SSMFbased Radio-over-Fiber link," 2018 International Topical Meeting on Microwave Photonics (MWP), Toulouse, 2018, pp. 1-4.doi: 10.1109/MWP.2018.8552895

[14] M. U. Hadi, P. A. Traverso, G. Tartarini, O. Venard, G. Baudoin and J. Polleux, "Digital Predistortion for Linearity Improvement of VCSEL-SSMF-Based Radio-Over-Fiber Links," in IEEE Microwave and Wireless Components Letters. doi:

10.1109/LMWC.2018.2889004

[15] Hadi, M.U., Nanni, J., Traverso, P.A., Tartarini, G., Venard, O., Baudoin, G., Polleux, J.L.: Linearity improvement of VCSELs based radio over fiber systems utilizing digital predistortion. Adv. Sci. Technol. Eng. Syst. J. 4(3), 156-163 (2019). doi: 10.25046/aj040321 [16] F. Fuochi, M.U. Hadi, J. Nanni, P.A. Traverso, G. Tartarini, "Digital predistortion technique for the compensation of nonlinear effects in radio over fiber links", 2016 IEEE 2nd International Forum on Research and Technologies for Society and Industry Leveraging a better tomorrow (RTSI), pp. 1-6, 2016

[17] Hadi, M.U. Nonlinearities Diminution in 40 Gb/s 256 QAM Radio over Fiber Link via Machine Learning Method. Preprints 2019, 2019090031 (doi:

10.20944/preprints201909.0031.v1).

[18] N. Aslam, K. Xia and M. U. Hadi, "Optimal Wireless Charging Inclusive of Intellectual Routing Based on SARSA Learning in Renewable Wireless Sensor Networks," in IEEE Sensors Journal, vol. 19, no. 18, pp. 8340-8351, 15 Sept.15, 2019. doi: 10.1109/JSEN.2019.2918865 